\title{
THE FUNCTIONS OPERATING ON HOMOGENEOUS BANACH ALGEBRAS ${ }^{1}$
}

\author{
BY MISHA ZAFRAN
}

Communicated by Elias Stein, May 20, 1977

In this note, we announce a negative solution to the "dichotomy problem" in the context of homogeneous Banach algebras. We begin with the following notation. Let $A(\mathrm{~T})$ denote the algebra of absolutely convergent Fourier series, and let $C(\mathrm{~T})$ be the class of all continuous functions on $\mathrm{T}$. Let $B$ be a semisimple, self-adjoint Banach algebra with maximal ideal space $\mathrm{T}$. We view $B$ as an algebra of continuous functions on T. $B$ will be called homogeneous provided the following two properties hold:

(1) For every $a \in \mathrm{T}$, the mapping $f(x) \rightarrow f(x+a)$ is an isometry of $B$ into itself.

(2) For every $f \in B$, we have

$$
\lim _{a \rightarrow 0}\|f(x+a)-f(x)\|_{B}=0 .
$$

$B$ will be called strongly homogeneous provided we also have

(3) For every integer $k$, the operator $f(x) \rightarrow f(k x)$ maps $B$ into itself and is of norm 1 .

It is well known that only analytic functions operate on $A(\mathrm{~T})$ (see [1] or [4, Chapter 6]). Clearly, all continuous functions operate on $C(T)$. We have the following "intermediate" result:

THEOREM 1. There exists a strongly homogeneous Banach algebra B satisfying the following two properties:

(a) $A(\mathrm{~T}) \varsubsetneqq B \varsubsetneqq C(\mathrm{~T})$.

(b) Nonanalytic functions operate on $B$.

The question solved by this result arose naturally in the study of the operational calculus of $A(\mathrm{~T})$ (see [1] and Chapter 6 of [4]). For some previous results related to this question, we refer the reader to [2] where the problem is specifically posed, and to [3].

We now indicate our construction of $B$. Let $\psi \in P$, the class of trigonometric polynomials. An admissible representation for $\psi$ is defined as an expansion of $\psi$ in the form $\psi=\Sigma_{k=1}^{n} a_{k} \psi_{k}$, where $\psi_{k} \in P$, and $\left\|\psi_{k}\right\|_{\infty} \leqslant 1,1 \leqslant k \leqslant n$. Define 


$$
\|\psi\|=\inf \sum_{k=1}^{n}\left|a_{k}\right| \log \left(\left\|\psi_{k}\right\|_{A(T)}+e^{2}\right),
$$

the infimum taken over all admissible representations $\psi=\Sigma_{k=1}^{n} a_{k} \psi_{k}$. It is not difficult to verify that $\|\cdot\|$ is an algebra norm on $P$ and $\|\psi\|_{\infty} \leqslant\|\psi\| \leqslant$ $3\|\psi\|_{A(T)}$, for all $\psi \in P$. Let $B$ denote the completion of $P$ under the norm $\|$.

Then $B$ is a strongly homogeneous Banach algebra on $\mathbf{T}$ and $A(\mathbf{T}) \varsubsetneqq B \varsubsetneqq$ $C(T)$. In fact, if $\psi \in B$, then $\{\hat{\psi}(n)\}$ is in the Lorentz sequence space $l_{2,1}$. Property (b) of Theorem 1 is an immediate consequence of the following result:

THEOREM 2.

$$
\sup _{\substack{\psi \in B \\ \psi \text { real } \\\|\psi\| \leqslant 1}}\left\|e^{i r \psi}\right\| \leqslant C_{1}|r|^{3 / 2} \exp \left(C_{2}|r|^{1 / 2}\right),
$$

for $|r| \geqslant 1$. Here $C_{1}$ and $C_{2}$ are absolute constants.

The above estimate is obtained by decomposing an admissible representation for a real trigonometric polynomial in a suitable manner. This permits us to exploit the "cancellation" properties of the logarithmic function used in the definition of $\|\cdot\|$. Detailed arguments will appear elsewhere (see [5]).

\section{REFERENCES}

1. H. Helson, J.-P. Kahane, Y. Katznelson and W. Rudin, The functions which operate on Fourier transforms, Acta Math. 102 (1959), 135-157.

2. Y. Katznelson, Calcul symbolique dans les algèbres homogènes, C. R. Acad. Sci. Paris 254 (1962), 2700-2702.

3. Y. Katznelson and P. Malliavin, Analyse harmonique dans quelques algèbres nomogènes, Israel J. Math. 5 (1967), 107-117.

4. W. Rudin, Fourier analysis on groups, Interscience, New York, 1967.

5. M. Zafran, The dichotomy problem for homogeneous Banach algebras (submitted for publication).

\footnotetext{
DEPARTMENT OF MATHEMATICS, STANFORD UNIVERSITY, STANFORD, CALIFORNIA 94305
} 